\title{
Craft Stick Beams
}

\author{
Alan K. Karplus \\ Western New England College
}

\section{Keywords:}

Beams, Bend Tests, Composite Structures

\section{Prerequisite Knowledge:}

\section{The student should}

1. know what a craft or popsicle stick is and that it can be bent and broken,

2. be familiar with fiberboard and/or cardboard and that it can be bent and torn apart, and

3. know that sticks of wood and fiberboard can be glued together to form beams of specified dimension.

\section{Objective:}

This exercise is to provide an phenomenological "hands-on" experience that shows how geometry can affect the load carrying capacity of a material used in construction how different materials have different failure characteristics, and how construction affects the performance of a composite material.

The student will learn to:

1. Fabricate different types of layered beams such as
A. Built-up beams of a single material, and
B. Composite beams of a mixture of materials,

2. Test these layered beams to determine how and where the beams fails,

3. Use knowledge gained from 1 and 2, to design a layered beam that will fail in a predicted manner, and

4. Capture a knowledge of the effects of lamination, adhesion, and geometry in layered beam construction on beam strength and failure location.

\section{Equipment and Supplies:}

1. Forster Craft Sticks, (Forster Inc. Wilton, ME) or popsicle sticks, fiberboard sheets from backs of pads of paper

2. Large Size Paper Cutter

3. Micrometer or Vernier Caliper to measure sample thickness

4. Safety Glasses

5. Glue - Elmer's Glue AU, Bordon, Inc. Columbus, $\mathrm{OH}$ is suggested, but any other glue can be used. In fact, glue type can become a variable. Be sure to use the same glue for ALL samples in a given test.

6. Clamps for holding glued craft sticks (an anvil capable of applying load over the complete craft stick is necessary for quality results)

7. Test Apparatus with three point loading fixture: Instron Load Frame with Load verses Deflection plot output capability or Hydraulic Tester and gage to read "on-the-run" the first maximum load. 
8. Data Sheet (see recommended samples below)

9. Magnifying glass

10. Computer spreadsheet if desired

\section{Procedure:}

Overview:

Step I is to make built-up beams. One, two ,three, and four craft sticks are used to make a series of unglued beams and a series of glued beams. Step II is to make fiber sticks cut from the backings of pads of paper and make fiber stick built-up beams: a series of unglued fiber stick beams and a series of glued fiber stick beams. Step III is to test each beam in the four series and make a record of the results of the mechanical testing so that composite beams can be designed. Step IV is to design series of composite beams with fiber sticks and craft sticks, and to prepare hypotheses that predict which layer in a composite beam will fail first and wherein the layer the failure will occur. Step V is to test each beam in a series of composite beams to accept or reject an hypothesis.

\section{Sample Preparation:}

The craft sticks are used as they come from the package. The fiber board needs to be cut to siie with a large paper cutter. A paper cutter minimizes distortion and breakage. The craft sticks are close to 0.088 by 0.364 by 4.488 inches in size while fiber boards from legal pads found were 0.073 inches thick. While 0.073 inches is less than 0.088 inches the thinner fiber board sticks were used due to legal pad availability.

\section{Sample Gluing}

The task of placing glue on the beams is significant. Select and use the same glue for all series needing glue. A good approach is to use Elmer's Glue All and a small brush to quickly lay a bead of glue which is spread with the brush, and if three craft sticks are to be joined, place glue on two craft sticks and place them together, rub them about and realign the sticks as one beam and place the composite beam into the clamp. Next apply the clamp force as soon as possible. Be sure to check alignment after clamping to be assured that all members are parallel. Makeup the series of beams you plan to evaluate, select the materials needed to build them, and build them, being sure to keep notes along the way.

To assist the experimenter in keeping good records, a beam series is defined as a group of samples which have common features. Four sticks are chosen because four trail sticks have the same height as the width of one craft stick. The series of Built-Up Beams follows:

\section{Wooden Craft Sticks}

a. Series I: First a craft stick alone, and then two, three, and four craft sticks stacked together, and

b. Series II: Two, three, and four of the wooden craft sticks are glued together on their wide flat faces as built-up beams.

2. Fiber stick beams are developed when fiber board as fiber sticks of the same thickness and size of the craft stick are used in place of the wooden craft sticks. The selections are:

a.. Series III: First a fiber stick alone, and then two, three, and four fiber sticks are stacked together, and

b. Series IV: Two, three, and four fiber sticks glued together on the wide flat faces as a built-up beam.

Test Activity Overview:

The fist step is to create reference data for comparison purposes. This will be the "benchmark" to which all subsequent findings are compared. Record failure loads in a table for one, then two, three, and four trail sticks placed together on their wide flat faces unglued (Series I), and loaded to find the first maximum failure load as the 
beam is loaded to failure. Failure occurs when the load carried by the beam decreases after reaching a maximum. Remember, the load is applied at a right angle to the wide flat face of the craft stick and uniformly across the width as shown in Figure 1. A plot of load against beam deformation can be made while the testis underway. Review of the plot may show jumps which can be due to material failure or debonding of glue that indicate significant changes in the ability of the beam to carry load. A loss of load signals a failure. These kinds of observations will be seen in the glued beams such as series II.

Testing - Phase I:

Two three point type beam test units are available: One is similar to an Instron machine under position control when the load rate is specified in inches per second and a plot of load against deflection is obtained. The second is an hydraulic jack setup which could be prepared as a clamp when curing the glued beams and as a device for obtaining maximum failure load for each beam loaded to failure. When craft sticks are used each, prepare the three point loading apparatus so the bottom supports are 4.00 inches apart and the third load is applied in the center or 2.00 inches from each end of the bottom supports. Kundu and Wickman (1) present an approach to making a mechanical tester.

Place abeam in the three point test fixture - two load edges on the bottom and one on the top makes three points. Be sure the load is applied perpendicular to the wide face of the craft sticks on the outside surfaces of a manufactured beam. Apply load until the beam fails. Failure is identified as the load indicator rises and then falls off as the beam is no longer able to support load. For the unglued beams sliding of the components relative to the upper stick is often noticeable, especially when noting the alignment at the end of a beam.. Use of a magnifying glass will help. Check the beam under the load points, on the free ends and under the center applied load. LISTEN and you may hear a noise as the beam or glue fails, too. Place on the data sheet the maximum load found just before the FIRST failure occurs. Continue this process for all beams in a series. Remember to make notes about the noises heard and the changes seen in the beam (i.e., in series IV with the three layered beam, did the fiber beam in the center layer crush?).

Series I: First a single stick beam and then built-up beams made from two, three, and four craft sticks should be tested and the first maximum load prior to failure recorded in the table. Written notes on the data sheet about the response of each beam to its three point bending evaluation are recommended. For example, for the single craft stick the maximum load carried is 12 pounds, the beam broke in the middle under the applied load and the beam splintered. In addition, written comments on a visual inspection of each beam before, during and after test can be helpful. Since there are many beams under evaluation, please label each beam in a series (series I - beam 1, etc.) and make notes before the next beam is loaded. Figure 2 shows the load deformation plot for one, two, three, and four layered glued craft sticks. The jumps present failures which often can be heard as a' snap' when the testis run.

\section{Composite Beams:}

All composite beams will be made with layers that are glued together. The naming of composite beams is used to specify beam stick layering. One or more fiber sticks is substituted for one or more of the wooden craft sticks when a composite beam is prepared. A scheme for designating wood sticks (W) and fiber sticks (F) components is created. If the first letter denotes the top surface and top member of the composite beam, then the following combinations may prove interesting: Series V which includes FW and WF, Series VI with members FWF, WFW, Series VII with members WFFW, FWWF, WFWF, and FWFW are suggested.

Hypothesis formation:

Each student should make an hypothesis which addresses which layer in the layered structure of a WFW composite beam will fail first and will it be crushed or splinter. The hypothesis should be formulated based on the findings 
from testing single material beams of wooden craft stick beams and fiber stick beams. The previously collected information can enhance your knowledge and is to be used to make an hypothesis for composite beam performance. For example, in series V, the first hypothesis is that the fiber stick (F) in the middle of a three layer composite beam (WFW) will be the first to fail and will it fail in the center of the beam layer beam by crushing or splintering, is made. Future hypotheses can address the expected location in the beam and the expected types of failures in WFFW, WFWF, and FWFW beams.

Testing- Phase II:

Composite beam load tests are performed to determine the effect of material arrangement for fabricated composite beams. The first hypothesis is for the WFW beam, and this hypothesis should be tested prior to testing other composite beams. Once confidence has been gained in predicting that the fiber stick will fail first and the failures will be due to separation within the fiber stick given that the glue between layers does not fail, new composite arrangements can be addressed. Sometimes failures will occur because of gluing technique and not because of materials arrangement. Remember the hypothesis presented above. When evaluating the series $\mathrm{V}$, three layered composite beam WFW be sure to look at the data from series I-IV and see what beam configuration is the strongest. How did the hypothesis fare? Was it accepted or rejected? The fiber layer in WFW usually fails directly under the applied load and is crushed.

It is always possible to check the current hypothesis a second time. This verification activity is accomplished by preparing a second sample of the beam from this series, but be sure the procedure used to make, glue, and clamp the beam is the SAME as before. Variation will be present, but the trend should be clear.

The experimenters will soon discover that making composite beams from layers of sticks which are glued together needs an expertise developed for glue application and clamping. The amount of clamping force can alter the quality of end product. Often the glue is not the weakest link and the beam material will fail first. However, uniform application of glue to the surfaces to be joined is important as otherwise premature failures may occur.

As multiple layered composite beams are made careful observation of beam layer failure may show a dramatic change or jump in load on a load against deformation graph that is made while the beam is under test. In addition, astute observation by a student may catch the layer destruction action when one member of the glued composite beam fails. As the load applied to a beam increases a careful observer can see the reaction on the beam (splinters can be formed, fiber beams can be crushed and separate from their glued outer surfaces, and sometimes the composite beam separates in the glued region at one end between the wood stick and the fiber stick or maybe within the fiber itself)! This information should be recorded and used when the next series of composite beams is designed and hypotheses are made for where the failure will occur.

Results Review:

Table 1- Sample Data at 0.01 inches per second Loading Rate: Pounds at Failure Load

\begin{tabular}{rllcccr}
\multicolumn{1}{l}{ Series } & I & II & III & I v & VII & S-2 \\
Layers :1 & 10.1 & ----- & 1.7 & ---- & 29.5 & 19.5 \\
2 & 18.2 & 37,4 & 2.0 & 6.6 & 44.2 & 58.5 \\
3 & 29.3 & 78.8 & 4.5 & 14.0 & 27.1 & 108.9 \\
4 & 35.4 & 90.9 & 4.6 & 21.3 & 28.3 & 197.6
\end{tabular}

Series I is craft sticks without glue.

Series II is craft sticks glued with Elmer's Glue All. 
Series III is fiber sticks without glue.

Series IV is fiber sticks glued with Elmer's Glue All.

Series VII is a 4 layer composite as follows for Layer 1 designation WFFW, Layer 2 designation FWWF, for Layer 3 designation WFWF, and Layer 4 designation FWFW.

Series S-2 is solid birch cut to thickness of layers designated (see Comments below).

From Table 1 data it is seen that the unglued craft sticks in series I carry more load than the fiber sticks in series III and that gluing sticks to double the beam height in a built-up beam can triple (two layers of craft sticks or fiber sticks) load carrying capacity. Built-up fiber stick beams appear not gain appreciable load carrying capacity after 3 layers if unglued, but that gluing makes a tremendous difference and increases the load carrying capacity over a single stick by seven or more times. The four layered unglued beams are not as good in carrying load as the two member glued beams. In series VII the combination WFWF is not as good as FWFW, and FWWF is best, but not as strong as four layers of glued craft sticks. From Figure 3 which is a plot of series II, series IV and series S-2 it can be seen shows the difference between the glued stick beams and solid wood beams of thickness similar to the layered beams. In all cases the center of the beam resists the transverse shear load on the beam which is greatest it the center of the cross-section at mid-span as presented by Hibbler (2). In all cases the solid wood samples were superior to the laminated beams.

\section{Comments:}

Extension of the concepts learned includes several initiatives which you and/or your students may want to follow: 1. A change in glue is possible. If this is done, be sure all sets of beams use this same glue so that one kind of glue can be compare to another. DAP Weldwood Carpenter's Glue, DAP, Inc., Dayton, OH Titebond Wood Glue, Franklin Chemical Industries, Columbus, $\mathrm{OH}$ and Dap Weldwood Hobby>n Craft Glue, DAP, Inc., Dayton, $\mathrm{OH}$ are a few that have been tried. Ask yourself a question - Is the glue weaker or stronger than the wood and/or the fiberboard used? Usually the glue is stronger and fails when the wood and/or fiber sticks change shape under load.

2. A set of solid wood beams with thickness of one, two, three, and four craft sticks with the depth and length the same as the craft sticks could be prepared. This should be done carefully because cutting small pieces the size of craft sticks can be dangerous. Seek the help of a craftsperson who knows how to do this type of work.

3. Different treatments could be given to the beams prior to testing. Painting or coating with a penetrating preservative can change attributes. Even wrapping the beams in tissue and painting with airplane dope will simulate a built-up wing type structure. You select the options!

4. A comparison of recorded information for white birch by Tsoumis (3) often referred to as Paper Birch, can provide some engineering calculations. The Wood Handbook (4) presents for paper birch with 12 percent moisture content a modulus of rupture of 12300 psi and a shear strength parallel to the grain of 1210 psi. These properties can be compared to values found for loads listed in Table 1, above, under column S-2 for the solid wood beams cut to order. Equations for the calculations tier Higdon (5) are available to students of engineering who have studied Mechanics of Materials. In addition, the Wood Handbook provides some reference to the "basic stresses" for structural members while reference information on the strength of glued joints is scare.

5. A set of sample of other materials can be used. Plastic materials and appropriate bonding agents some of which might be solvents, can be considered. A feature of this approach is that several student teams can be selected, given same size geometries and appropriate bonding agents, sent to independently build uniform material beams or composite material beams and all brought together for testing and/or data analysis. 


\section{References:}

1. Kundu, N.K. and Wickman, J.L., An Affordable Material Testing Device, NASA Conference Publication 3304, National Educators' Workshop: New 94, November 1994, pp.201-209.

2. Hibbler, R. C., Mechanics of Materials, Macmillan Publishing Company, New York, NY, 1991, Ch 7.

3. Tsoumis, G., Wood As A Raw Material, Pergamon Press, N.Y.,NY, 1968, p.229

4. The Forest Products Laboratory, Wood Handbook, Agricultural Handbook 72, Superintendent of Documents, U.S. Printing Office, 1955

5. Higdon, A. Et.al., Mechanics of Materials, 4th edition, John Wiley \& Sons, Inc. N. Y., NY, 1985

\section{Acknowledgments:}

The author received assistance over the 1995 summer from Robert Chhugani, a Western New England College incoming Senior Mechanical Engineering student and from Mathew and Jeff Kolb, brothers and local Springfield, MA highschool students, Their endeavor and comments were valuable and greatly appreciated.

\section{ALAN K. KARPLUS}

Alan K. Karplus is Professor of ME at Western New England College, Springfield, Massachusetts. He has a BSME from Tufts, an MS from Iowa State Univ. and a PhD from Colorado State Univ. He has been involved with the Freshman Engineering, Junior and Senior Mechanical Engineering Laboratory, teaches Materials Science and supervises Senior Projects. He is a member of ASME, ASEE and ASM International. 